UCRL-ID-116054

\title{
Voltage Controlled MESFET Pulse Shape Generator
}

Scott C. Burkhart

October 26, 1994

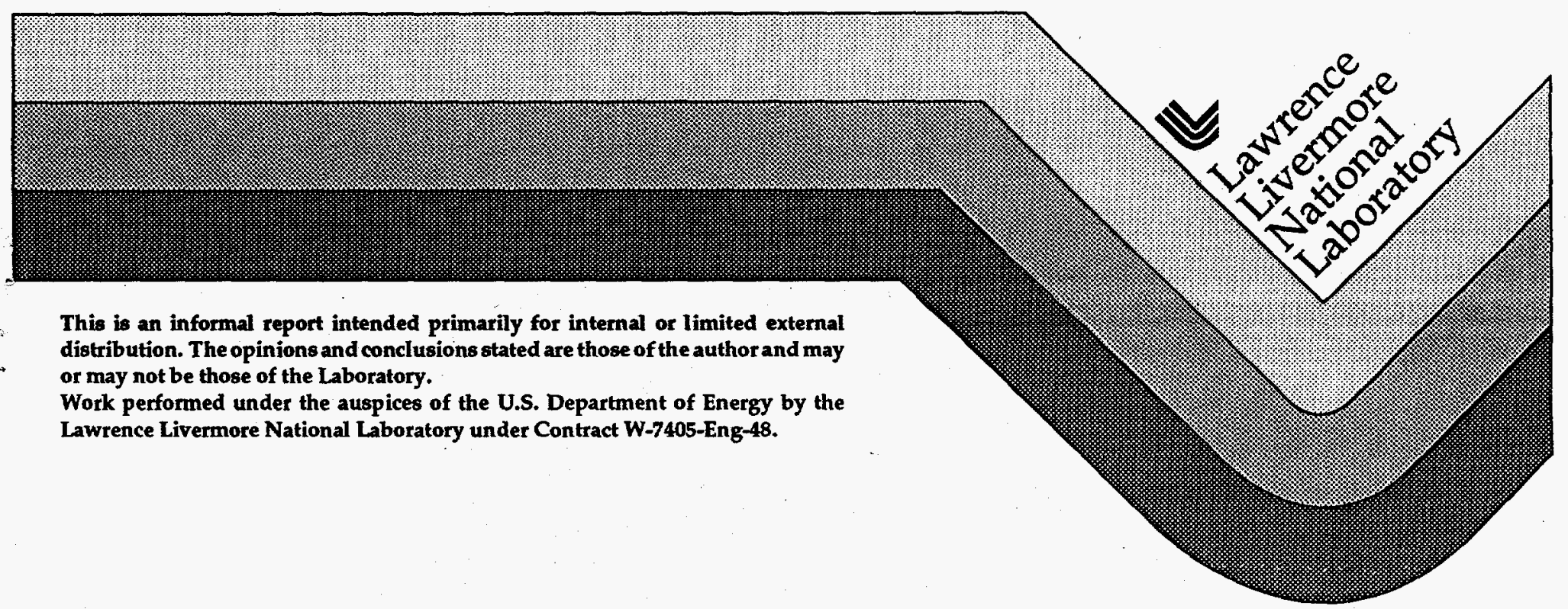




\section{DISCLAIMER}

This report was prepared as an account of work sponsored by an agency of the United States Government. Neither the United States Government nor any agency thereof, nor any of their employees, make any warranty, express or implied, or assumes any legal liability or responsibility for the accuracy, completeness, or usefulness of any information, apparatus, product, or process disclosed, or represents that its use would not infringe privately owned rights. Reference herein to any specific commercial product, process, or service by trade name, trademark, manufacturer, or otherwise does not necessarily constitute or imply its endorsement, recommendation, or favoring by the United States Government or any agency thereof. The views and opinions of authors expressed herein do not necessarily state or reflect those of the United States Government or any agency thereof. 


\section{DISCLAIMER}

Portions of this document may be illegible in electronic image products. Images are produced from the best available original document. 


\section{ABSTRACT \\ Voltage Controlled MESFET Pulse Shape Generator}

S. C. Burkhart

A programmable pulse shape generator capable of producing pulse shapes for Nova and Beamlet has been designed and simulated using the circuit code SPICE. The design utilizes power MESFETS, which are commonly used in microwave amplifiers. The pulse shape is varied by setting a bias voltage on each in a chain of MESFETS with a $200 \mathrm{ps}$ temporal resolution. The electrical pulse then drives an integrated electro-optic modulator similar to what is on Beamlet. Pulse shapes 22 and 25, used on Nova, have been generated by this design.

\section{Introduction}

In the NIF 90 day study, the Front End pre-conceptual design included the capability for temporal pulse shaping of each beamlet. The proposed design included proven devices from the Beamlet MOR design such as the integrated electro-optical amplitude modulator, and the design also included an electrical programmable shape pulse generator to drive the modulator. While I asserted that such a generator could be built, such a prototype had yet to be designed or even simulated using electrical circuit simulation codes. However, in the study, the basic architecture was described as a chain of high speed microwave transistors, triggered in sequence, to impart current impulses of varying amplitude upon the charged pulse line. The size of the current impulses was to be determined by a bias voltage, which would allow rapid re-programming of the pulseshape. I have now designed a feasible circuit and simulated the it using the well known circuit simulation code SPICE. In the simulations, I have demonstrated the pulse shaping circuit in its basic form. 


\section{Circuit Design}

The circuit is designed as shown in the simplified Fig. 1. The two transmission lines shown consist of the pulse line (top) and the trigger line (bottom). The pulse line is connected every $200 \mathrm{ps}$ to ground through a Gallium-Arsenide Metal-Semiconductor Field Effect Transistor (GaAs MESFET) which is a widely used microwave frequency transistor. All of the MESFETs are biased in the off state, which is typically $-2 \mathrm{~V}$ to $-4 \mathrm{~V}$ relative to the MESFET source (the lower terminal). For the devices used here, NEC NE900200 MESFETs, the turn off voltage is nominally $-3.5 \mathrm{~V}$. To initiate operation, a pulse of $270 \mathrm{ps}$ FWHM at $75 \mathrm{~V}$ is launched on the trigger line and propagated past each of the MESFETs. Each MESFET gate is fed $4 \mathrm{~V}$ of the trigger pulse through the tap resistor connected to the trigger line, and the $4 \mathrm{~V}$ pulse is transmitted through the blocking capacitor directly to the MESFET gate. This is the point where the programmability of this design exists, because the MESFET is turned on in proportion to the initial charge voltage of the gate bias. If the gate bias was initially $-8 \mathrm{~V}$, the $4 \mathrm{~V}$ pulse from the trigger line has no effect since the gatesource voltage never exceeds the cut off voltage of $-3.5 \mathrm{~V}$. On the other hand, if the bias voltage was initially $-4 \mathrm{~V}$, the trigger pulse raises the gate to $0 \mathrm{~V}$, taking it from saturation to the linear region, and pulling the pulse line down from $10 \mathrm{~V}$ to about $0.9 \mathrm{~V}$. When current is extracted from the pulse line, a voltage pulse or wavelet travels in both directions, the left traveling wave going to the termination resistor, with the right traveling wave becoming a part of the shaped pulse which is being constructed from these wavelets.

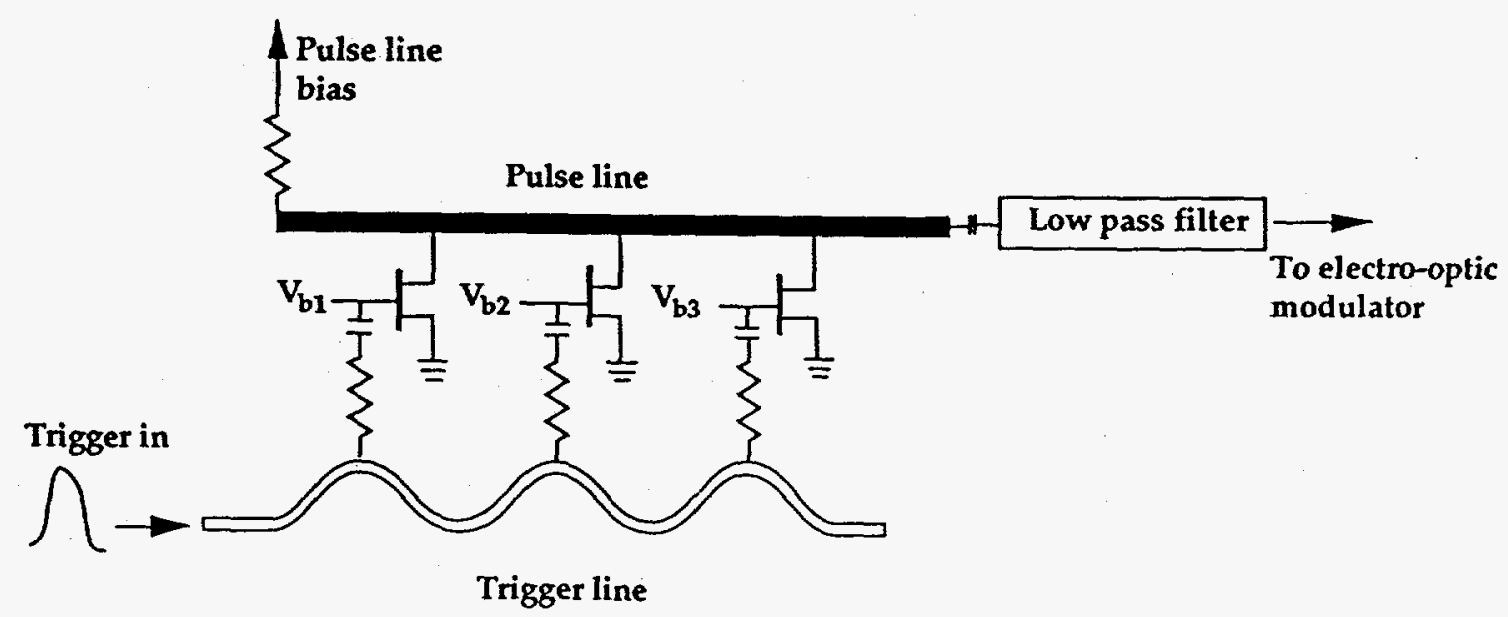

Figure 1. Three sections of the pulse shaping system. Microwave transistors switch facsimilies of the trigger pulse onto the Pulse line. 
The shaped pulse is built up as shown in Fig. 2. The first wavelet becomes the rising edge of the initial shaped pulse. The trigger line delay is 420 ps between MESFETs as compared to the $200 \mathrm{ps}$ along the pulse line. Thus, after each wavelet is added to the shaped pulse, it propagates entirely past the next MESFET, which is triggered at such a time to add on the next part of the shaped pulse. In this way, the pulse is assembled until the desired pulse shape has been generated. The pulse propagates through a DC blocking capacitor, and through a maximally linear phase (Bessel) low pass filter to remove the ripples from the wavelet addition. The final shaped pulse propagates to an integrated electro-optical modulator, identical in purpose to the Nova pulseshaping Pockels cell, and identical to the modulator used on Beamlet.

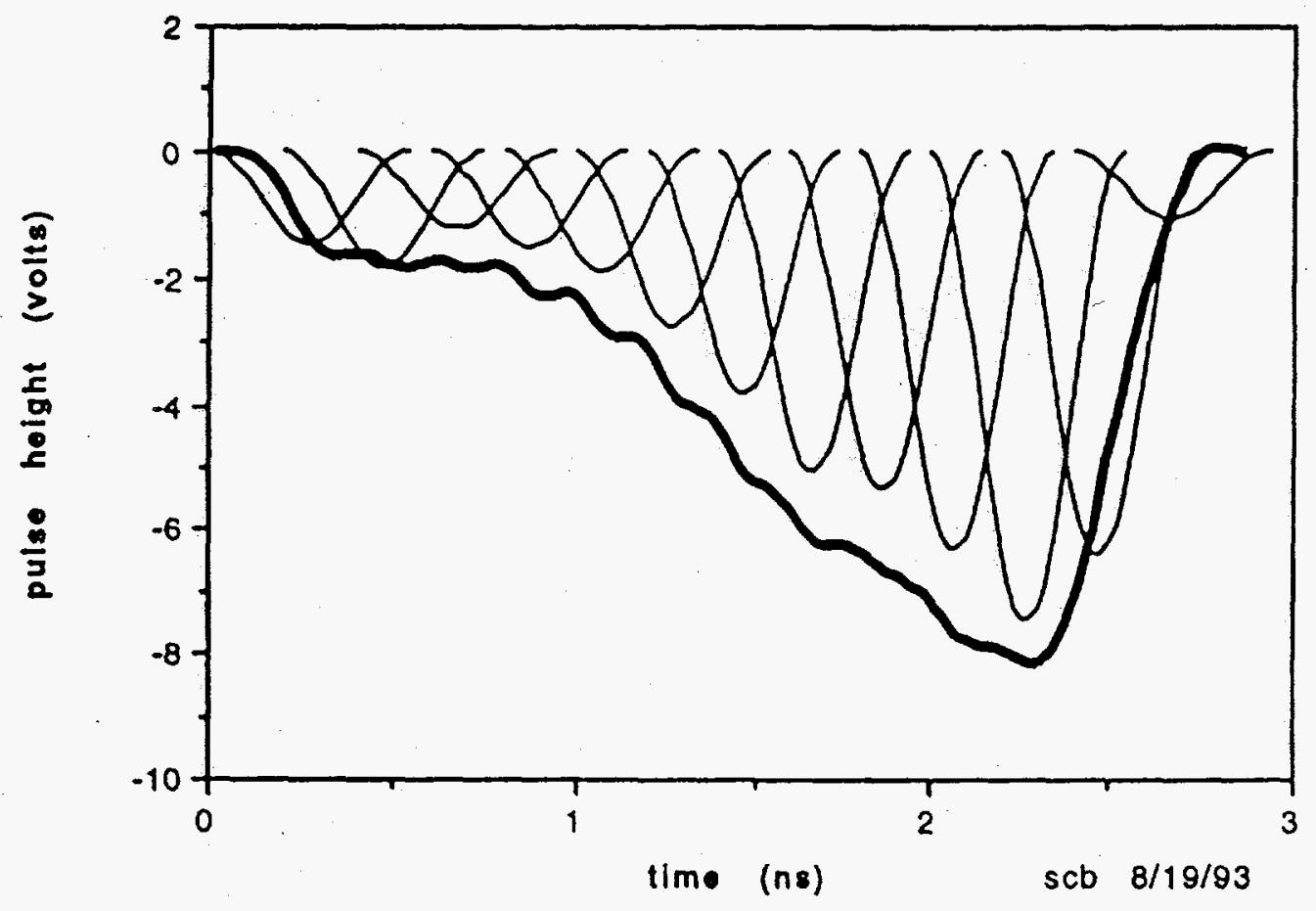

Figure 2. The final shaped pulse is constructed from scaled and delayed replicas of the trigger pulse. 


\section{Circuit Modeling}

The circuit was modeled as shown in Fig. 1, using the complete circuit shown in Figures 3 and 4. The NEC NE900200 devices were designed for use in microwave power amplifiers at frequencies up to $20 \mathrm{GHz}$, and have a low drain-source capacitance, and a low drain-ground capacitance which is required in this application. Simulation of this device was done using a combination of behavioral and physical models. There exists at least five different equivalent circuit models created by various researchers starting with the Curtice model in 1980. The model which I used was developed by Statz et al., in 1987.

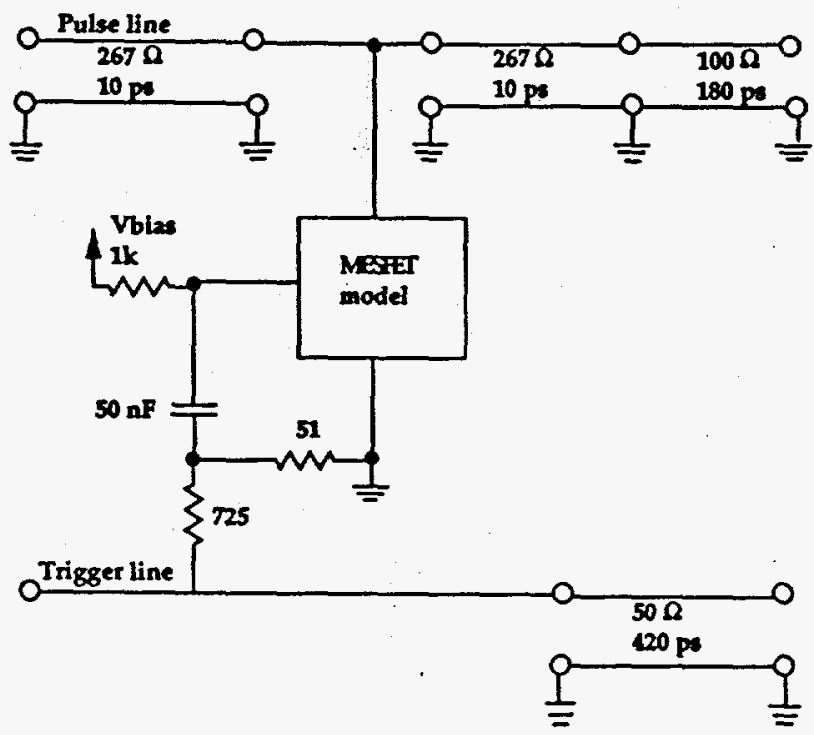

Figure 3. The pulse shaping circuit consistu of this sub-circuit cascaded many timer. Each of these dreuits controls a 200 pe section of the shaped pulse.

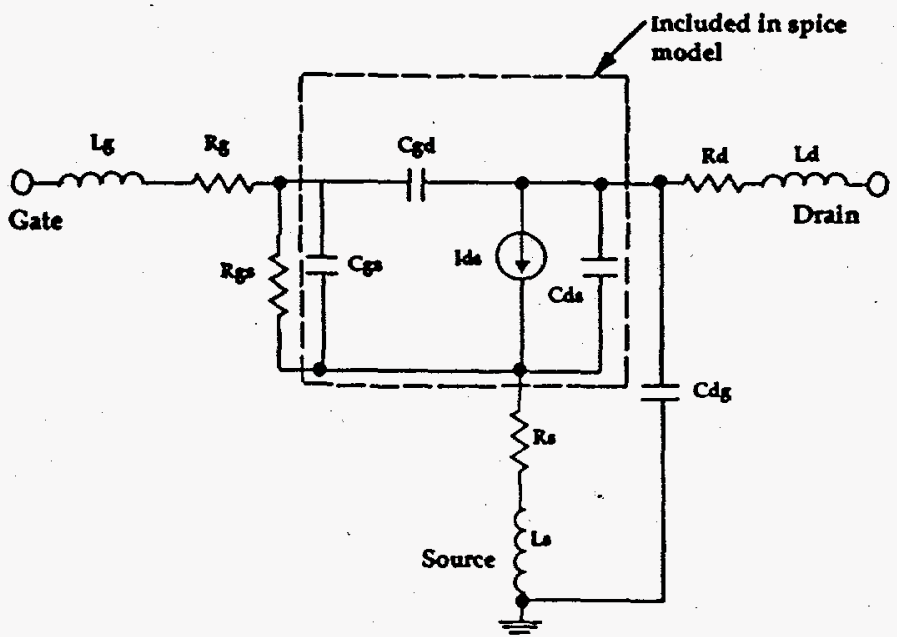

Figure 4. MESFET model used in SPICE. The formula used to determine Ids is that given by eq. 1. Cds, $\mathrm{Cdg}$ and $\mathrm{C}_{\mathrm{gs}}$ are deternined from complicated relations involving $V_{g B}, V_{g d}$, and several other of the MESFET model parametern. 
It has several advantages over the basic Curtice model, such as improved accuracy for modeling the drain-source current (Ids) for large gate-source voltages (Vgs), and more accurage modeling of the gate-drain and gate-source capacitance ( $\mathrm{Cgd}$ and $\mathrm{Cgs}$ ). For the Statz model, the drain-source current Ids is given by:

$$
I d s=\underbrace{\frac{\beta\left(V_{g s}-V_{r o}{ }^{2}\right)}{1+b\left(V_{g s}-V_{r o}\right)}}_{A}[\underbrace{\frac{1-\left(1-\alpha V_{d s}\right)^{3}}{3}}_{B}](\underbrace{1+\lambda V_{d s}}_{c})
$$

Briefly, part A of the equation models the square law dependence of Igs for small $\mathrm{V}_{\mathrm{gs}}-\mathrm{V}_{\mathrm{TO}}$ (where $\mathrm{V}_{\mathrm{TO}}$ is the MESFET pinch-off voltage). For larger $\mathrm{V}_{\mathrm{gs}}-\mathrm{V}_{\mathrm{TO}}$, the dependence becomes nearly linear. Part B models the transition from Vds $=0$ to where the region charge carrier saturation velocity occurs at $V_{\mathrm{ds}} \sim 1 \mathrm{v}$. Part $\mathrm{C}$ of the equation accounts for the resistive behavior during saturated operation. As I did not have any devices to characterize, I relied upon the manufacturer's numbers and SPICE defaults to set the parameters as shown in Table 1.

Table 1. MESFET Parameters used in the Statz Model in SPICE

$\begin{array}{lcllll}\text { Parameter } & \text { Value } & & \text { Parameter } & \frac{\text { Value }}{1} & \\ \beta \beta & .012 & \mathrm{~V}^{2} & \mathrm{Rd} & 1.8 & \mathrm{Ohm} \\ \mathrm{V}_{\mathrm{TO}} & -3.5 & \mathrm{~V} & \mathrm{Ls} & 18 & \mathrm{pHy} \\ \mathrm{b} & 0.3 & \mathrm{~V}^{-1} & \mathrm{Rs} & 0.9 & \mathrm{Ohm} \\ \alpha & 2.0 & \mathrm{~V}^{-1} & \mathrm{Rgs} & 4.0 & \mathrm{k}-\mathrm{ohm} \\ \lambda & 0.1 & \mathrm{~V}^{-1} & \mathrm{Cdg} & 0.25 & \mathrm{pF} \\ \mathrm{Lg} & 12 & \mathrm{pHy} & \mathrm{Cgd} & 0.084 & \mathrm{pF} \\ \mathrm{Rg} & 0.49 & \mathrm{Ohm} & \mathrm{Cgs} & 1.61 & \mathrm{pF} \\ \mathrm{Ld} & 12 & \mathrm{pHy} & \mathrm{Cds} & 0.13 & \mathrm{pF}\end{array}$

Several additional circuit considerations were made to successfully generate the pulses which have been obtained. For example, the trigger pulse is attenuated by the tap resistors as it propagates along the trigger transmission line, requiring smaller resistors for the downstream MESFETs. A general result for this sort of tapped transmission line is that each subsequent resistor is $Z_{0} / 2$ less than the previous one, where $Z_{0}$ is the characteristic impedance of the transmission line. This insures that the identical current is sent to each of the MESFETS. A wave is reflected back from each tap resistor junction, but the magnitude 
is small enough not to retrigger any of the upstream MESFETS. The other modification to this circuit was to account for the gate-drain and drain-ground capacitance. The drain-ground capacitance is $0.25 \mathrm{pF}$, which is enough to cause a significant reflection of a wavelet passing by the MESFET drain connection. Added to that is the gate-drain capacitance of $.08 \mathrm{pF}$, which approximately adds to the drain-ground capacitance depending upon the gate waveform. Compensation for this shunt capacitance is done by reducing the pulse transmission line capacitance local to the drain connection. This was accomplished with a $10 \mathrm{ps}, 267 \Omega$ section of the pulse transmission line on either side of the drain. With the MESFET capacitance included, the 20 ps section approximated a 100 ohm transmission line for minimal pulse distortion and reflection.

\section{Modeling Results}

For the purposes of demonstrating the capability of this circuit, I chose to build Pulse 22, which is a frequently used drive on Nova. Pulse 22 is shown in Figure 5, scaled to 7 volts, with an overlay of the SPICE electrical output. Seven volts was chosen to satisfy the input requirements of the electro-optic modulator. The simulation was performed on a Macintosh IIfx, and required approximately 21 minutes of CPU time to obtain $6 \mathrm{~ns}$ of operation. While Pulse 22 as shown in Fig. 5 is really the master oscillator $1 \mathrm{w}$ optical pulse, not the electrical pulse, I could just as easily have generated the electrical pulse.

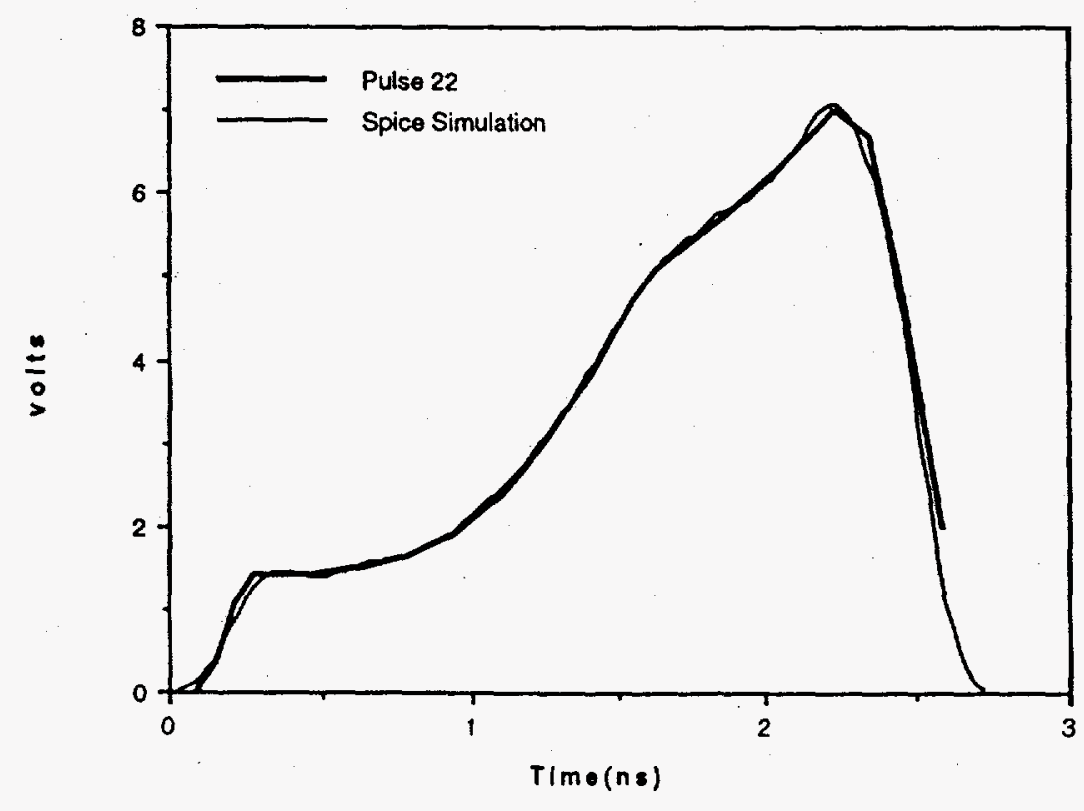

Figure 5. Nova Pulse 22 scaled to $7 \mathrm{~V}$ and compared to the Pulse from the simulated pulse generation circuit. 
There is some crosstalk between the individual wavelets, thus one cannot independently adjust each wavelet's amplitude without affecting the amplitude of the following wavelet. The initial settings for the MESFET bias voltages were obtained using an independent analysis of square pulse amplitude vs. bias voltage, followed by a spline interpolation to translate the Pulse 22 amplitude to bias voltages. The second and subsequent iterations were done by comparing the result to Pulse 22, and calculating new bias values. The interpolation and other analysis was accomplished using MATHCAD.

\section{Conclusion}

A programmable pulse shape generator for Nova style pulse shapes has been designed and simulated using the electrical circuit simulation package SPICE. The results are very encouraging, with a demonstrated capability to produce Nova Pulse 22 to a high degree of accuracy. There is no fundamental barrier to making such a pulse generator for use on the National Ignition Facility. In fact, the longer time scales on the NIF pulse will ease the high speed requirements of the pulse shape generator allowing the use of less expensive components. The next step will be to build a prototype circuit for initial testing on Beamlet and Nova.

\section{References}

1. J. M. Golio, Microwave MESFETs \& HEMTs, Norwood, MA: Artech House, 1991, pp. 128-138.

2. W. R. Curtice, "A MESFET Model for Use in the Design of GaAs Integrated Circuits," IEEE Trans. on Microwave Theory Tech., Vol. MTT-28, pp. 448-456.

3. H. Statz, P. Newman, I. W. Smith, R. A. Pucel, H. A. Haus, "GaAs FET Device and Circuit Simulation in SPICE," IEEE Trans. on Electron Devices, Vol, ED-34, pp. 160-169. 